\title{
Les formations ouvertes et à distance
}

Open and Distance Learning

Las formaciones abiertas y a distancia

\section{Chantal D'Halluin et Michel Loonis}

\section{OpenEdition}

\section{Journals}

Édition électronique

URL : http://journals.openedition.org/ries/2755

DOI : $10.4000 /$ ries. 2755

ISSN : 2261-4265

Éditeur

Centre international d'études pédagogiques

Édition imprimée

Date de publication : 23 septembre 1999

Pagination : 107-116

ISSN : $1254-4590$

Référence électronique

Chantal D'Halluin et Michel Loonis, "Les formations ouvertes et à distance », Revue internationale d'éducation de Sèvres [En ligne], 23 | 1999, mis en ligne le 23 septembre 2002, consulté le 30 avril 2019. URL : http://journals.openedition.org/ries/2755 ; DOI : 10.4000/ries.2755 


\title{
Les formations ouvertes et à distance
}

\author{
Chantal D'Halluin
}

Michel Loonis

\begin{abstract}
Open and Distance Learning
How can Open and Distance Learning meet the ever growing public demand ? The authors first explain the basic concepts of Open and Distance Learning and some of the risks and answer the question through two examples of higher education degrees organised at the University of Lille.
\end{abstract}

\section{Las formaciones abiertas y a distancia}

¿Cómo la formación abierta y a distancia puede satisfacer la demanda cada vez mayor del público? Después de definir los conceptos básicos y los peligros de la formación abierta y a distancia, los autores responden a esta pregunta a partir de dos ejemplos provenientes de un centro universitario de Lille, ambos relacionados con la preparación a los concursos de educación superior.

\section{FOAD : pourquoi ?}

Les attentes du public par rapport à la formation ont sensiblement changé au cours des dernières années : les adultes en formation acceptent de moins en moins les méthodes scolaires de transmission du savoir. Ils refusent les programmes de formation étendus qui les obligent à travailler sur des contenus qu'ils maîtrisent déjà et sont demandeurs de formations parfaitement adaptées à leurs besoins, à leurs acquis et à leur disponibilité. Ils souhaitent maîtriser leurs parcours de formation, choisir les modules dont ils ont besoin et apprendre au moment qui leur convient. Qu'ils soient salariés ou en recherche d'emploi, ils refusent de plus en plus les déplacements, coûteux en temps et en énergie, et souhaitent adapter les modes de formation à leurs conditions de vie et de travail.

La généralisation, au moins sur les lieux de travail, de l'usage des NTIC en font des médias désormais acceptés pour se former. 
Comment mieux adapter la formation aux besoins, aux contraintes, aux possibilités des individus et des organisations ? C'est à cette question qu'essaie de répondre un organisme de formation en mettant en œuvre des formations ouvertes et à distance, c'est-à-dire en adaptant à chaque individu et/ou organisation :

- la formation aux contraintes socio-économiques,

- le contenu de formation aux besoins et aux objectifs,

- le rythme de formation au rythme d'apprentissage,

- le mode de formation aux modes d'apprentissage,

tout en privilégiant une dimension collective de la formation.

\section{Qu'est-ce qu'une FOAD?}

Dès 1993, la Délégation à la formation professionnelle (ministère du Travail et de l'Emploi) a défini les formations ouvertes et à distance comme étant «des dispositifs de formation s'appuyant pour tout ou partie sur des apprentissages non présentiels, en autoformation, ou avec tutorat, à domicile, dans l'entreprise et/ou en centre de formation. Ce qui caractérise fondamentalement les formations ouvertes et à distance, c'est la souplesse de leur mode d'organisation pédagogique et donc leur plus grande accessibilité, comparées aux formations "traditionnelles". Centrées sur l'individu, les formations ouvertes n'excluent pas le formateur, ni le groupe; ni éventuellement les autres membres du collectif de travail. Elles peuvent alterner des séquences individuelles et collectives. Les objectifs généraux des formations ouvertes et à distance sont de faciliter l'accès à la formation professionnelle et de promouvoir de nouveaux modes d'apprentissage. »

Cette définition fait aujourd'hui l'objet d'un consensus tant au niveau des instances européennes qu'internationales même si les appellations diffèrent (flexible training, flexible learning, Open and Distance Learning, etc.)

Une caractéristique des formations ouvertes et à distance est l'articulation de l'espace et du temps de formation. Ainsi, seront considérées comme "ouvertes» les formations auxquelles formateurs et apprenants participent :

- soit en même temps, mais dans des espaces différents,

- soit dans le même lieu mais à des moments différents,

- soit enfin à des moments et dans des lieux différents.

Ces nouvelles temporalités et ces nouveaux lieux de la formation entraînent, dans leur sillage, un renouvellement radical de la conception des dispositifs, des approches pédagogiques (autoformation individuelle et collective, apprentissage organisationnel), des méthodes, techniques et bien sûr des outils de formation. L'ensemble des modes d'action pédagogique s'en trouve également affecté. La règle des "trois unités " du théâtre classique (unité de temps, de lieu et d'action), qui régit de fait la majorité des formations présentielles (horaire fixe, salle permanente, mode d'action pédagogique unique), se trouve ainsi remplacée par celle des "trois flexibilités " des formations ouvertes : temps modulables, espaces variables, modes d'action différenciés. 
Au-delà de cette caractéristique qui prend en compte les flexibilités, le concept d'" ouverture "-remet en question la qualité des systèmes de formations existants et induit la conception de nouveaux systèmes de formation plus adéquats aux personnes et aux organisations.

\section{La mise en œuvre d'une FOAD?}

La prise en compte des caractéristiques de souplesse et de transformation d'une formation ouverte et à distance entraîne un renouvellement radical de la conception des dispositifs (formation individualisée, alternance, centre de ressources, formation à distance, etc.), des approches pédagogiques (individualisation, autoformation, tutorat, apprentissage coopératif), des ressources et des technologies. La mise en place de formations ouvertes et à distance bouleverse l'organisation traditionnelle du système éducatif, en plaçant l'apprenant au cœur du dispositif comme acteur de sa formation. La notion de contrat pédagogique et didactique est introduite, le dispositif est lui-même situé dans un environnement social et technologique.

En fait, un tel dispositif met en jeu trois niveaux :

- " micro », prenant en compte ce qui se passe chez les acteurs : apprenants, formateurs, administratifs, technologues ;

- " méso », prenant en compte ce qui se passe dans le dispositif luimême : sa cohérence, sa régulation, son adaptation à la demande de formation des individus et des collectifs, son évolution d'un point de vue didactique, pédagogique, structurel ;

- «macro ", prenant en compte ce qui se passe pour le dispositif, dans l'organisme de formation, dans l'environnement social et politique.

La mise en œuvre de formations ouvertes et à distance exige des conditions spécifiques radicalement différentes de la mise en place de formations conventionnelles. Ces conditions portent sur les personnes ressources, les modalités d'organisation, les ressources pédagogiques.

\section{Les personnes ressources}

Parmi les personnes ressources, la plus importante est bien évidemment le formateur, mais le travail en équipe est plus que jamais indispensable ; le formateur doit intégrer son travail dans celui d'une équipe administrative, pédagogique, technologique.

Le formateur n'est plus le seul détenteur du savoir mais il est expert du domaine, animateur du groupe, pilote de l'action, organisateur de la communication pédagogique...

Il convient plus d'identifier des fonctions que des personnes, les fonctions pouvant être remplies par une ou plusieurs personnes suivant leurs compé- 
tences. Parmi les fonctions, nous identifions : une fonction «concepteur de ressources»; une fonction "formateur» (il est expert de la matière, conduit l'apprentissage de chaque apprenant, anime la communication pédagogique, évalue les apprentissages) ; une fonction «relais pour la technologie» (rendre accessible les outils, mettre à disposition les ressources) ; une fonction «lien permanent» (transmission des informations, mise en relation des acteurs, traitement des imprévus).

\section{Les ressources médiatisées}

En formation ouverte et à distance, les apprenants travaillent principalement à partir de supports pédagogiques médiatisés. Leur conception nécessite un savoir-faire technologique, pédagogique et didactique.

Les finalités, objectifs, contenus, pré-requis, conditions d'évaluation, publics doivent être clairement définis ; la méthode pédagogique doit être explicite pour les formateurs, comme pour les apprenants; les choix didactiques doivent être explicites pour les formateurs qui prennent le cours en charge et pour les apprenants au cours de l'apprentissage. La référence à un ou à des modèles d'apprentissage doit également être explicite.

La médiatisation implique aussi le choix du média : quels sont les médias adaptés en fonction du contenu, de la méthode pédagogique, du contexte, du public cible?

Mais quelles que soient les ressources utilisées, le formateur devra très souvent adapter le contenu de la formation aux objectifs des apprenants. Il est très important que les ressources soient organisées en "briques » de base qui serviront à constituer des parcours individualisés à partir desquels il est possible de personnaliser les itinéraires de formation.

\section{Les modalités de la formation}

La FOAD n'est pas synonyme de formation individuelle. Même dans le cas de formation individualisée, la référence à un collectif est un gage de réussite de l'apprentissage. La contractualisation des temps collectifs et des temps individuels de formation est une étape obligatoire.

De la même façon, la FOAD ne signifie pas isolement ; les interactions, entre formateur et apprenant(s) d'une part, et entre apprenants d'autre part, sont déterminantes pour le processus d'apprentissage. La séparation spatiale et/ou temporelle induit la médiatisation des interactions ; la communication devient intentionnelle contrairement au présentiel où elle est spontanée. Aussi les interactions n'auront lieu que si le cadre de la formation les prévoit par une gestion rigoureuse du temps et de l'espace, par la planification de certaines d'entre elles, en veillant à l'accessibilité des outils nécessaires. 
Dans une formation présentielle, la comptabilisation du temps de formation est chose simple, pour le formateur comme pour les apprenants : c'est la somme des heures passées ensemble. Il en va tout autrement en FOAD où les temps du formateur, de chaque apprenant, de la formation ne se recouvrent pas forcément. Il est donc nécessaire d'établir des règles strictes de comptabilisation des temps du formateur, de chaque apprenant, de la formation, et de veiller à une cohérence globale entre les différentes comptabilisations, notamment d'un point de vue financier.

\section{Une ingénierie pour les FOAD?}

Par sa définition même, une formation ouverte et à distance se doit d'être souple, adaptable, mais en même temps des contrats sont passés sur le temps, sur les ressources, sur l'utilisation des outils. Il est très important que chacun tienne son rôle et remplisse ses obligations. Le formateur est en quelque sorte sous tension, entre les deux pôles que sont la flexibilité et la rigueur. Il en ressort que la mise en place d'un système ouvert n'est pas sans risque, que la rigueur peut aboutir à une industrialisation et une déshumanisation de la formation, que les rôles des uns et des autres peuvent se parcelliser et se figer. Les modes de formation peuvent se rigidifier : dans une formation de groupe, par exemple, on arrive à ne plus prendre en compte la spécificité de chaque indivi$\mathrm{du}$. Les formations individualisées deviennent alors individuelles et la formation à distance renforce l'isolement.

Pour veiller à ce que les deux caractéristiques de la FOAD (souplesse et adaptabilité à l'environnement) soient maintenues tout au long du processus de formation, pour garder la rigueur et la cohérence nécessaires à la réussite du dispositif, un pilotage conséquent et continu, tant sur le plan de l'ingénierie de formation que sur le plan de l'ingénierie pédagogique est indispensable.

\section{L'apprentissage coopératif : une pratique à développer}

Les activités coopératives dans l'apprentissage contribuent à donner une dimension collective aux formations individualisées et à rompre l'isolement dans les formations à distance.

Par référence aux modèles pédagogiques dominants, on peut dire que l'apprentissage coopératif :

- s'oppose au mode transmissif - un apprentissage significatif, une compréhension en profondeur s'établissent après conversation, débat, argumentation entre différentes personnes ;

- ne s'identifie pas à une pédagogie de groupe ou à une pédagogie active- 
dans un apprentissage coopératif, chacun est acteur à part entière ;

- ne s'identifie pas à une communication, celle-ci est un pré-requis pour la coopération.

L'apprentissage coopératif privilégie les communications interpersonnelles et le débat collectif. Il s'agit de travailler ensemble, de créer quelque chose de nouveau - à travers la coopération et la collaboration -, de rassembler les compétences individuelles au bénéfice de la communauté, le tout dépassant chaque partie.

Apprendre de façon coopérative implique des échanges entre pairs, des interactions, des changements de rôles suivant les besoins.

Dans ce modèle, l'apprenant est central. Il assume la responsabilité de son apprentissage. Il organise ses activités d'apprentissage. L'apprenant est autonome : c'est un apprentissage individuel, comme résultat d'une interaction collective. Les interactions entre personnes entraînent une modification, une réorganisation des structures de connaissances et de compréhension de chacun. Par la communication et l'interaction, le processus cognitif, en grande partie implicite, devient explicite, grâce à la verbalisation et à l'écrit.

Le développement d'activités coopératives d'apprentissage est particulièrement important dans une formation d'adultes à distance. D'une part, les adultes préfèrent se focaliser d'emblée sur des problèmes à résoudre plutôt que sur des concepts abstraits sans fondements significatifs pour eux. Ils apprennent 112 mieux en faisant et ils utilisent leur expérience sociale et professionnelle dans leur processus d'apprentissage. D'autre part, le public de formation à distance est un public isolé, contraint d'apprendre seul si de telles activités ne sont pas possibles. Des outils de communication spécifiques, des environnements sont nécessaires pour permettre cet apprentissage coopératif. Ils permettent d'abaisser les barrières de temps et d'espace qui inhibent l'interaction dans la pratique de l'enseignement à distance. Ils réduisent les contraintes, tout en laissant de la liberté aux apprenants, sans leur donner d'obligations supplémentaires.

Les groupes de discussion, favorisant la construction de concepts chez l'apprenant, la résolution collective de problèmes ou d'études de cas, la pédagogie de projet avec la conception coopérative d'objets, sont des exemples significatifs d'apprentissage coopératif. 


\section{Étude de cas Deux exemples de FOAD}

Le centre université-économie d'éducation permanente (CUEEP), créé en 1969, est un institut de l'université des sciences et technologies de Lille, spécialisé dans la formation permanente des adultes. Environ vingt mille personnes par an s'inscrivent dans ses filières de formation qui vont de l'illettrisme à l'entrée à l'université. Le CUEEP a également une grande activité en formation de formateurs. Il propose des parcours individualisés s'appuyant sur des formations en groupe, individualisées, à distance, en atelier de pédagogie personnalisée. Pour les institutions et les entreprises, l'ingénierie recouvre une activité de création de systèmes complets de formation, de conception de formation et d'adaptation des pratiques de formation.

Le laboratoire de recherche Trigone, rattaché au CUEEP, est composé de trois équipes dont les champs d'investigation sont complémentaires : méthodologie générale, analyse des dispositifs et pratiques éducatives (MEGADIPE), ingénierie des formations ouvertes (OPEN), nouveaux outils pour la communication et l'éducation (NOCE). Le développement de l'environnement du travail coopératif assisté par ordinateur est l'un des axes de recherche commun aux équipes NOCE et OPEN.

\section{La préparation au DAEU}

Le diplôme d'accès aux études universitaires (DAEU) est un diplôme spécifique à la formation d'adultes qui confere à ceux-ci les mêmes droits que le baccalauréat.

Dans la région Nord-Pas-de-Calais, la préparation aux DAEU A (littéraire) et $B$ (scientifique) est organisée en système modulaire et unités capitalisables. Pour les différentes matières obligatoires et optionnelles, une vingtaine de modules de soixante heures, de trois niveaux (pré-DAEU, DAEU, spécialisation), sont proposés par les six universités de la région Nord-Pas-de-Calais qui travaillent en collaboration. La préparation est ainsi délocalisée dans vingt-deux centres universitaires ou centres liés par convention. Le candidat détermine avec l'aide d'un conseiller en formation la structure du DAEU la mieux adaptée à son projet. Un positionnement de départ est effectué pour les matières comportant plusieurs niveaux. Une procédure de validation des acquis par unité est possible.

Plusieurs modes de formation sont proposés en formation présentielle ou à distance:

- le groupe modulaire : groupe d'environ vingt personnes encadré par un formateur. Ce mode de formation fondé sur une pédagogie active est en place depuis plus vingt ans et a toujours sa raison d'être;

- la formation matière individualisée : collectif de quinze apprenants encadré par un formateur. Le parcours est individualisé en fonction des acquis, des objec- 
tifs, du rythme, du mode d'apprentissage de chaque individu. Les modalités de la formation sont contractualisées avec le formateur. Ce mode permet de gérer efficacement les contraintes dues au travail posté, les déplacements professionnels mais aussi les rythmes et les modes d'apprentissage différents des apprenants. Le formateur est expert de la discipline et gestionnaire des activités d'apprentissage, il essaie en particulier d'organiser des séquences d'apprentissage collectives par petits groupes;

- la formation modulaire à distance : formation plus individuelle pour répondre aux contraintes d'horaires et de rythmes des individus. Le contenu de chaque module est référencé, identique au présentiel. Les documents sont des outils multimédias avec une prédominance forte du papier, la plupart des apprenants ne disposant pas d'outils informatiques. L'intégration des différents médias et les cours en ligne sont aujourd'hui, dans ce dispositif, du domaine de l'expérimentation. Le suivi par le formateur est individuel et à distance par courrier et téléphone. Un secrétariat pédagogique sert de lien permanent entre apprenants et formateur en dehors des permanences téléphoniques. Le public de ce mode de formation peut être caractérisé comme isolé et contraint ;

- la formation individualisée à distance est calquée sur la formation modulaire à distance; les modalités sont identiques mais le parcours est individualisé.

Depuis le démarrage de la formation à distance, un public se trouve contraint de suivre un enseignement à distance sans le souhaiter : ce sont les personnes qui désirent suivre un module qui n’est pas programmé dans le centre le plus proche de leur domicile, faute de candidats en nombre suffisant. Il s'agit là d'une contrainte structurelle qui n'est pas toujours bien acceptée par la personne, contrairement aux contraintes citées plus haut qui sont des contraintes individuelles. Pour répondre à ce public, les universités ont mis en place un nouveau mode de formation:

- le groupe modulaire virtuel (GMV) est une formation de groupe tant sur le plan du rythme que de l'organisation. On y retrouve les caractéristiques du groupe modulaire. Le GMV met en relation des personnes distantes grâce aux NTIC. Elles communiquent entre elles par audioréunion, visioconférences ou partage d'écran et d'applications informatiques. Les conditions sont satisfaisantes tant au point de vue relationnel (on s'entend, on se voit, on partage des documents en temps réel), que pédagogique (la multiplicité des outils de communication synchrone et asynchrone accessibles permet une véritable interaction pédagogique), que financier (les coûts des équipements et des communications diminuent, la fiabilité augmente);

- l'autoformation tutorée est accessible librement, sur conseil ou sur prescription ; elle se déroule en centre de ressources animé par un tuteur méthodologue qui aide les apprenants dans leur démarche d'apprentissage. Le temps de formation est libre ou contractualisé suivant les conditions d'accès. Le formateur détermine la formation après évaluation, il valide la formation en fin de parcours et intervient si nécessaire sur demande.

Les ressources pédagogiques médiatisées sont utilisées dans les différents modes de formation et accessibles dans les centres universitaires de ressources éducatives. 


\section{Le diplôme général de pédagogie de l'université du Caire}

Le diplôme général de pédagogie (DGP) est un diplôme de l'Institut des études et des recherches pédagogiques de l'université du Caire. Ce diplôme existait préalablement à l'établissement de la convention entre l'université du Caire et l'université de Lille I. L'objectif est de mettre à disposition, pour la formation des enseignants égyptiens des écoles de langue française et des écoles gouvernementales expérimentales, les recherches françaises en matière de pédagogie, de didactique et d'utilisation pédagogique des NTIC. Ceci afin de valoriser le diplôme en évitant de juxtaposer une formation universitaire française et en tenant compte du système éducatif égyptien.

Les enseignements du DGP - option langue française - sont organisés conjointement par l'université du Caire et l'université de Lille I.

Les enseignements dispensés en français sont assurés, sous la responsabilité de l'université de Lille I, par Lille I même, par l'IUFM du Nord-Pas-de-Calais et par des personnels du centre français de culture et de coopération (CFCC) de l'ambassade de France en Egypte.

La délivrance du DGP - option langue française - est placée sous la responsabilité de l'université du Caire. L'évaluation des enseignements donnés en français est effectuée par l'université de Lille I dans les conditions générales définies dans l'organisation du diplôme. Les examens ont lieu à l'université du Caire suivant les conditions générales du DGP. L'ensemble des cours en français représentent environ $40 \%$ du total des enseignements.

Les enseignements assurés par les universitaires en France se font entièrement à distance. Un tutorat local est organisé sur l'année par le CFCC, à raison de deux heures par semaine pour chaque cours. Il faut noter le rôle important des tuteurs, aidant les étudiants à se familiariser avec les nouveaux outils d'information et de communication, ainsi que ce nouveau mode de formation. Ces tuteurs apportent une aide méthodologique dans l'organisation du travail, pour la réflexion et la compréhension.

Les supports de cours sont de type hypertexte. Ils sont directement accessibles en ligne sur le réseau de l'internet; pour des raisons financières et techniques, ils sont consultables également en réseau local.

L'objectif est de proposer des documents aussi interactifs que possible, incluant une partie informative et des indications de travail précises qui invitent l'auditeur à compléter le cours ou à le mettre en pratique par des activités d'analyse, de lecture, de production de supports, etc. Une attention toute particulière est également accordée aux moyens de communication permettant d'accompagner et d'aider le groupe dans son travail.

Les interactions directes entre les étudiants et les enseignants de Lille sont régulières; elles se font en asynchrone (mél., courrier, fax) et en synchrone (utilisation de Netmeeting pour une communication écrite, d'un serveur audio pour la 
communication orale). Le campus virtuel du CUEEP (laboratoire Trigone) structure ces interactions dans un environnement de travail coopératif, c'est-à-dire que l'on trouve réunis les documents de cours, des documents réalisés par les membres du groupe disponibles comme documents de travail et de réflexion, les conversations entre les membres du groupe.

La création d'un diplôme de l'université de Lille I est envisagée : le diplôme universitaire d'études de pédagogie pour les enseignants en langue française.

Ce diplôme s'appuierait pour l'essentiel sur la formation du DGP et comprendrait trois types d'enseignements :

- les enseignements en langue française du DGP, organisés et validés sous la responsabilité des enseignants de Lille ;

- des unités qui s'appuient sur des enseignements du DGP dispensés en langue arabe. Ces enseignements seront complétés par des ressources en langue française et validés par la rédaction d'un mémoire dirigé à distance ;

- des enseignements complémentaires à distance suivis après l'obtention du DGP.

Pour ces deux derniers cas, le campus virtuel supportera le travail de groupe à distance.

Ce diplôme, créé à la demande des partenaires égyptiens, rencontre les préoccupations d'autres pays de la francophonie et pourrait être adapté aux conditions spécifiques de chacun d'entre eux.

\section{Le campus virtuel}

Nous avions besoin d'une véritable organisation pour supporter l'apprentissage flexible et à distance.

Parmi les NTIC, les systèmes informatiques de travail coopératif assisté par ordinateur ont démontré leur efficacité en tant que support d'activités coopératives liées à la réalisation de projets professionnels.

Dans le domaine de l'éducation, il s'agit de mettre en place un outil qui s'adapte aux exigences des différents contextes.

Les différentes recherches menées au sein du laboratoire Trigone, et par d'autres, nous ont amenés à la conception d'un campus virtuel capable d'accueillir une grande variété de modes d'apprentissage et d'organisation d'enseignements, tout en privilégiant l'appropriation et la construction des savoirs par la communication et la collaboration.

Le campus virtuel comprend deux aspects indissociables:

- l'organisation et l'environnement des formations ;

- la mise à disposition de contenus de formation, organisés en éléments de base, qui sont assemblés à la demande en des parcours individuels ou collectifs, en fonction des publics ciblés ou des demandes des utilisateurs. 